\title{
Functional Interaction between Pregnane X Receptor and Yes-Associated Protein in Xenobiotic-Dependent Liver Hypertrophy and Drug Metabolism
}

\author{
Taiki Abe, Ryota Shizu, Takamitsu Sasaki, Yuki Shimizu, Takuomi Hosaka, \\ Susumu Kodama, Atsushi Matsuzawa, and Kouichi Yoshinari \\ Division of Drug Metabolism and Molecular Toxicology (T.A., S.K., K.Y.) and Laboratory of Health Chemistry (T.A., A.M., K.Y.), \\ Graduate School of Pharmaceutical Sciences, Tohoku University, Aoba-ku, Sendai, Japan; and Laboratory of Molecular \\ Toxicology, School of Pharmaceutical Sciences, University of Shizuoka, Suruga-ku, Shizuoka, Japan (T.A., R.S., T.S., Y.S., T.H., \\ K.Y.)
}

Received April 3, 2019; accepted September 10, 2019

\begin{abstract}
Pregnane $X$ receptor (PXR), a xenobiotic-responsive nuclear receptor, plays key roles in drug disposition. PXR activation induces liver hypertrophy in rodents, but the molecular mechanism of this effect remains unclear, although the PXR-mediated induction of cytochrome P450s (P450s) is proposed to be involved. Since yes-associated protein (YAP), an effector protein of the Hippo pathway, functions as a transcriptional cofactor that controls organ size via TEA domain family members (TEADs) or other transcription factors, we investigated the functional interaction of PXR with YAP in liver hypertrophy and drug metabolism in this study. The treatment of mice with a PXR activator induced liver hypertrophy, promoted nuclear YAP accumulation, and increased the expression of YAP/TEAD target genes in the liver, suggesting the coactivation of PXR and YAP. Through chronological analyses of this in vivo model, no clear association between PXR-dependent liver hypertrophy and P450 induction was observed. In reporter assays, ligand-activated PXR enhanced YAP-mediated gene transcription, whereas YAP overexpression inhibited PXR-dependent gene transcription. No clear species
\end{abstract}

differences in these transcriptional interactions between humans and mice were observed. Furthermore, in human hepatocarcinoma and primary hepatocyte-like cells, YAP suppressed the expression of liver-enriched transcription factors, including hepatocyte nuclear factor $4 \alpha$, PXR, the constitutive androstane receptor, and their target genes. These results suggest that YAP is involved in PXRinduced liver hypertrophy and that YAP activation interferes with gene expression associated with various liver functions.

\section{SIGNIFICANCE STATEMENT}

We have investigated the functional interaction between PXR and YAP, an effector protein of the Hippo pathway. PXR plays central roles in various liver functions including drug metabolism, and the Hippo pathway and YAP regulate organ size through interacting with several transcription factors, including TEADs. Our results suggest that YAP is involved in PXR-mediated liver hypertrophy and that YAP activation interferes with the expression of liver-enriched transcription factors and thus drugmetabolizing enzymes.

\section{Introduction}

Pregnane X receptor (PXR) and constitutive androstane receptor (CAR), members of the nuclear receptor superfamily, are well-characterized xenobiotic-sensing transcription factors (Kliewer et al., 2002). These receptors play key roles in expression of a number of genes associated with drug disposition and energy metabolism (Kawamoto et al., 2000; Kodama et al., 2004; Yoshinari et al., 2010). Furthermore,

This study was supported in part by JSPS KAKENHI Grant Number JP17K08418 and a grant from the Takeda Science Foundation.

The authors declare that they have no conflict of interest.

https://doi.org/10.1124/jpet.119.258632. the activation of these receptors induces centrilobular hepatocyte hypertrophy and thus liver enlargement in mammals (Maronpot et al., 2010; Hall et al., 2012). However, PXR and CAR have completely different functions in hepatocyte proliferation. In rodents, various CAR activators, such as phenobarbital, induce hepatocyte proliferation and hepatocarcinogenesis (Butler, 1978; Williams and Whysner, 1996; Wei et al., 2000; Blanco-Bose et al., 2008; Hernandez et al., 2009; Tolson and Wang, 2010; Abe et al., 2017), while PXR activators do not. Recently, we reported that mouse PXR (mPXR) activation enhances xenobiotic- and growth factor-mediated hepatocyte proliferation by inhibiting FOXO3-dependent gene transcription in mice (Shizu et al., 2013, 2016). In addition, we demonstrated

ABBREVIATIONS: Ad, adenovirus; AML12, alpha mouse liver 12; CAR, constitutive androstane receptor; CITCO, 6-(4-chlorophenyl)imidazo[2,1-b] [1,3]thiazole-5-carbaldehyde O-(3,4-dichlorobenzyl)oxime; HNF4 $\alpha$, hepatocyte nuclear factor $4 \alpha ;$ hPXR, human pregnane X receptor; hYAP, human yes-associated protein; MOI, multiplicity of infection; mPXR, mouse pregnane $\mathrm{X}$ receptor; mTOR, mammalian target of rapamycin; mYAP, mouse yes-associated protein; P450, cytochrome P450; PCN, pregnenolone $16 \alpha$-carbonitrile; PCR, polymerase chain reaction; PXR, pregnane X receptor; siRNA, small interfering RNA; TCPOBOP, 1,4-bis[2-(3,5-dichloropyridyloxy)]benzene; TEAD, TEA domain family member; WT, wild type; YAP, yesassociated protein. 
that yes-associated protein (YAP), an effector protein of the Hippo pathway, plays a crucial role in CAR-dependent hepatocyte proliferation (Abe et al., 2018). Although the association of the induction of drug-metabolizing enzymes, including cytochrome P450s (P450s), with hepatocyte hypertrophy has been suggested (Maronpot et al., 2010), it remains unclear why PXR and/or CAR activation induces hepatocyte and liver hypertrophy.

Organ size is controlled by various factors associated with cell number, size, and density through multiple pathways, including the Hippo pathway and the mammalian target of rapamycin (mTOR) pathway (Lee et al., 2007; Lin et al., 2013). The former is evolutionally conserved among a number of species, including humans and rodents, and composed of mammalian sterile 20-like kinases 1 and 2, or MST1 and MST2, and large tumor suppressor kinases 1 and 2, or LATS1 and LATS2, in mammals. In the normal liver, the Hippo pathway is highly activated (Zhou et al., 2009; Song et al., 2010), and LATS1 and/or LATS2 thus phosphorylate YAP, a transcription cofactor that acts as an effector protein of this pathway, to retain and inactivate YAP in the cytoplasm (Lee et al., 2007; Lin et al., 2013). However, in liver tumors and other cancers, the Hippo pathway is often disrupted (Zender et al., 2006; Harvey et al., 2013). Under the latter conditions, nuclear levels of YAP are elevated, suggesting YAP activation and the Hippo pathway inactivation. Activated YAP regulates the TEA domain family member (TEAD)- and other transcription factor-mediated expressions of genes associated with cell proliferation and antiapoptotic genes (Guo and Teng, 2015).

Recently, we reported crosstalk between CAR and YAP (Abe et al., 2018) and demonstrated that the treatment of mice with the CAR ligand 1,4-bis[2-(3,5-dichloropyridyloxy)]benzene (TCPOBOP) promotes the nuclear accumulation of YAP in livers and that activated CAR augments YAP/TEAD-dependent gene expression. Furthermore, treatment with verteporfin, which disrupts the formation of the YAP-TEAD complex (Liu-Chittenden et al., 2012), prevented CAR-dependent liver enlargement in mice; these results suggest that CARmediated liver hypertrophy occurs, at least in part, through YAP/TEAD activation. However, whether PXR-mediated liver hypertrophy involves YAP/TEAD activation remains unclear.

In general, the expression of drug-metabolizing enzymes, including $\mathrm{P} 450 \mathrm{~s}$, is decreased in liver tumors and hepatoma cell lines (Rodríguez-Antona et al., 2002), but the underlying mechanism for this decrease remains unclear. P450s work as an important defense system against toxic substances and metabolize numerous xenobiotics, including food constituents, medicines, and residual pesticides. In the liver, many kinds of P450 genes are transcriptionally regulated by hepatocyte nuclear factor $4 \alpha(\mathrm{HNF} 4 \alpha)$, PXR, and CAR. Since YAP activation is involved in hepatocarcinogenesis and the formation of other tumors (Steinhardt et al., 2008; Xu et al., 2009; Wang et al., 2010; Zhang et al., 2011, 2014), we hypothesized that YAP activation affects PXR- and other transcription factor-dependent gene transcription to decrease P450 expression in liver tumors. Therefore, in this study, we investigated the functional crosstalk between PXR and YAP.

\section{Materials and Methods}

Materials. TCPOBOP, pregnenolone $16 \alpha$-carbonitrile (PCN), rifampicin, 6-(4-chlorophenyl)imidazo[2,1-b][1,3] thiazole-5-carbaldehyde $O$-(3,4-dichlorobenzyl)oxime (CITCO), and collagenase (type
IV) were obtained from Sigma-Aldrich (St. Louis, MO). Oligonucleotides were synthesized by Fasmac (Atsugi, Japan). Anti-histone-1 and anti-calreticulin antibodies were obtained from StressGen Biotechnologies (Victoria, BC, Canada) and anti-YAP antibody was obtained from Santa Cruz Biotechnology (Santa Cruz, CA). All other chemicals were of the highest grade available and were obtained from Wako Pure Chemical Industries (Osaka, Japan), Thermo Fisher Scientific (Waltham, MA), Sigma-Aldrich, or Promega (Madison, WI).

Animals and Primary Hepatocytes. Male C57BL/6 mice (approximately 8 weeks old; Charles River Laboratories Japan, Yokohama, Japan) were maintained in a temperature- and light-controlled room $\left(24^{\circ} \mathrm{C}, 12\right.$-hour light/dark cycles). Mice were intraperitoneally treated with PCN (100 mg/kg), TCPOBOP (3 mg/kg), or vehicle (corn oil, $20 \mathrm{ml} / \mathrm{kg}$ ) once a day for three consecutive days. The mice were sacrificed by cervical dislocation, and their livers were collected at 1,3 , 6 , and 9 days after the last treatment. The animal experiments were approved by the committees for animal experiments at Tohoku University and University of Shizuoka and conducted while following the guidelines for animal experiments at Tohoku University and University of Shizuoka.

Mouse primary hepatocytes were isolated from the liver by two-step collagenase perfusion as described previously (Seglen, 1976; Abe et al., 2017). Isolated hepatocytes were seeded in collagen type I-coated 24well plates (Corning, Corning, NY) at 1 or $2 \times 10^{5}$ cells/well and cultured in Williams' E medium (Thermo Fisher Scientific) supplemented with 10\% FBS (Sigma-Aldrich), ITS Premix (Corning), GlutaMAX (Thermo Fisher Scientific), and $100 \mathrm{nM}$ dexamethasone (Wako Pure Chemical Industries) for 4 hours. The hepatocytes were subsequently infected with adenovirus as indicated and treated with chemicals under serum-free conditions.

Cell Culture and Adenovirus Infection. Cells derived from the human hepatocellular carcinoma (HepG2), human embryonic kidney (293T), and African green monkey kidney (COS-1) were obtained from RIKEN BioResource Research Center (Tsukuba, Japan) and cultured in Dulbecco's modified Eagle's medium (Wako Pure Chemical Industries) supplemented with 10\% FBS, minimum Eagle's medium nonessential amino acids, and Antibiotic-Antimycotic (Thermo Fisher Scientific). The passage numbers of the cells used in this study ranged from 5 to 10 .

After 48-hour preculture, HepG2 cells were infected with each adenovirus, cultured for 48 hours, and then treated with $10 \mu \mathrm{M}$ rifampicin or vehicle (0.1\% DMSO) for an additional 24 hours under serum-free conditions. Alpha mouse liver 12 (AML12) cells (immortalized murine hepatocyte cell line) were purchased from American Type Culture Collection (Manassas, VA) and cultured as described previously (Shizu et al., 2016). After 24-hour preculture, the cells were infected with each adenovirus and cultured in serum-free medium for 48 hours and then treated with $10 \mu \mathrm{M}$ PCN or vehicle (0.1\% DMSO) for an additional 24 hours.

HepaRG cells, which are terminally differentiated primary hepatocyte-like cells, were purchased from Oriental Yeast (Tokyo, Japan). The cells were seeded in collagen type I-coated 48-well plates and cultured in HepaRG working medium (Thermo Fisher Scientific) for 6 hours. Then, the medium was changed to fresh HepaRG working medium. After 66 hours of culture, the cells were infected with the $\beta$-galactosidase-expressing adenovirus, Ad-LacZ, or dominant active human YAP (hYAP)-expressing adenovirus (Ad-hYAP-5SA) for 48 hours and then cultured with serum-free HepaRG induction medium (Thermo Fisher Scientific) containing $10 \mu \mathrm{M}$ rifampicin, $0.5 \mu \mathrm{M}$ CITCO, or vehicle (0.1\% DMSO) for an additional 48 hours.

Small Interfering RNA Transfection. HepG2 or AML12 cells were reverse transfected with $10 \mathrm{nM}$ ON-TARGETplus SMART Poolhuman YAP [hYAP-small interfering RNA (siRNA); GE Healthcare Dharmacon, Lafayette, CO]for HepG2, $10 \mathrm{nM}$ ON-TARGETplus SMART Pool-mouse YAP [(mYAP)-siRNA] for AML12, or ONTARGETplus Non-Targeting Pool (HepG2 and AML12) using Lipofectamine RNAiMAX (Thermo Fisher Scientific), cultured for 48 hours, and then treated with $10 \mu \mathrm{M}$ rifampicin (HepG2), $10 \mu \mathrm{M}$ 
PCN (AML12), or vehicle (0.1\% DMSO; HepG2 and AML12) for 24 hours.

Plasmid Preparation. p3A4 (CYP3A4 promoter-containing luciferase plasmid), mPXR-pTargeT (mPXR expression plasmid), human PXR (hPXR)-pTargeT (hPXR expression plasmid), mYAP-WTpTargeT (wild-type (WT) mYAP expression plasmid), and mYAP5SA-pTargeT (dominant active mYAP expression plasmid) have been described previously (Toriyabe et al., 2009; Yoshinari et al., 2012; Shizu et al., 2016; Abe et al., 2018). The hYAP expression plasmid was prepared as follows: hYAP cDNA was amplified by polymerase chain reaction (PCR) with the primer set indicated in Table 1 and inserted into the pTargeT plasmid (Promega). To prepare the hYAP dominant active form (hYAP-5SA), five serine residues (hYAP; S61, S109, S127, S164, and S397) were mutated to alanine residues using a KOD-PlusMutagenesis Kit (Toyobo, Osaka, Japan) with the primer set indicated in Table 1. To prepare the Flag-tagged mYAP-5SA (Flag-mYAP-5SApTargeT) and V5-tagged mPXR (mPXR-V5-pTargeT) plasmids, each plasmid was tagged at the $\mathrm{N}$ - or $\mathrm{C}$-terminus with the specific primers shown using the KOD-Plus-Mutagenesis Kit. The pTA-Luc control and TEAD-pTA plasmids were purchased from Signosis (Santa Clara, CA) and phRL-TK encoding Renilla luciferase was purchased from Promega.

Construction of Recombinant Adenovirus. The open reading frames of mYAP and hYAP were isolated from each expression plasmid (described previously) by PCR using specific primer sets (Table 1) and subcloned into the appropriate plasmids with the ViraPower Adenoviral Expression System, pENTR Directional TOPO Cloning, and pAd/CMV/V5-DEST Gateway Vector kits (Thermo Fisher Scientific) according to the manufacturer's protocols. One day before transfection, $293 \mathrm{~T}$ cells were seeded in 6-well plates (Sanplatec, Osaka, Japan) at $5 \times 10^{5}$ cells/well, and the plasmids that had been linearized with PacI (New England BioLabs, Ipswich, MA) were transfected into the 293T cells using FuGENE HD (Promega). The next day, the medium was replaced with fresh medium and the cells were cultured for an additional day. The cells were transferred to a 10$\mathrm{cm}$ tissue culture dish (Sanplatec), cultured until a cytopathic effect was observed on $80 \%$ of the visible region of the cells, and then the medium was replaced by fresh medium every 3 days. The cells and supernatants were collected, frozen at $-80^{\circ} \mathrm{C}$ for 30 minutes, and then thawed at $37^{\circ} \mathrm{C}$ for 15 minutes. This freeze/thaw cycle was repeated twice. To generate adenovirus at a higher titer, the $293 \mathrm{~T}$ cells were seeded at $3 \times 10^{6}$ cells $/ 10$-cm dish 1 day before infection, and $100 \mu \mathrm{l}$ of the crude viral lysate was added to the cells. Three days later, the cells and medium were harvested and subjected to the aforementioned freeze/thaw cycles twice and centrifuged. The obtained lysates were stored at $-80^{\circ} \mathrm{C}$ until use. The control adenovirus ( $\beta$-galactosidaseexpressing adenovirus, Ad-LacZ), hPXR-expressing adenovirus (AdhPXR), and Ad-mPXR-V5 have been described previously (Shizu et al., 2013, 2016).

Immunohistochemistry. Livers were fixed in $10 \%$ neutral buffered formalin (Wako Pure Chemical Industries). Paraffin-embedded liver sections were sliced into $4-\mu \mathrm{m}$-thick sections. The sections were stained by Morphotechnology (Sapporo, Japan) and image capture and acquisition were carried out as described previously (Shizu et al., 2016).

Quantitative Reverse Transcription PCR. Total RNA preparation, cDNA synthesis, and quantitative reverse transcription PCR using GoTaq qPCR Master Mix (Promega) were performed as described previously (Shizu et al., 2016; Abe et al., 2018). The specific primer sets used are given in Table 2. Relative mRNA levels were calculated by the $\Delta \Delta \mathrm{Ct}$ method using $18 \mathrm{~S}$ ribosomal RNA levels; $G A P D H$, encoding glyceraldehyde-3-phosphate dehydrogenase; or $A C T B / A c t b$, encoding $\beta$-actin, mRNA levels as references.

Reporter Gene Assay. COS- 1 cells seeded in 96-well plates at $1 \times$ $10^{4}$ cells/well were forward transfected with p3A4, TEAD-pTA, or the corresponding control plasmid, each expression plasmid and the control phRL-TK plasmid using FuGENE HD. Twenty-four hours later, the cells were treated with $10 \mu \mathrm{M}$ PCN, $10 \mu \mathrm{M}$ rifampicin, or vehicle $(0.1 \% \mathrm{DMSO})$ for 24 hours in serum-free medium. Reporter activities were determined as described previously (Shizu et al., 2016; Abe et al., 2018).

Western Blot Analyses. Liver nuclear extracts were prepared by using NE-PER Nuclear and Cytoplasmic Extraction Reagents (Thermo Fisher Scientific). Liver microsomal fractions were prepared as follows: the livers from three mice $(0.1 \mathrm{~g}$ each) were pooled and minced on ice and then homogenized in $1 \mathrm{ml}$ of $0.25 \mathrm{M}$ sucrose, $1 \mathrm{mM}$ EDTA, and $10 \mathrm{mM}$ Tris-HCl (pH 7.4). The homogenates were centrifuged at $9000 \mathrm{~g}$ for 20 minutes, and the resultant supernatants were centrifuged at $105,000 \mathrm{~g}$ for 1 hour. The obtained pellets were washed three times with the homogenizing buffer and suspended in $10 \%$ glycerol, $1 \mathrm{mM}$ EDTA, and $10 \mathrm{mM}$ Tris- $\mathrm{HCl}$ ( $\mathrm{pH}$ 7.4). Western blot analyses were performed as described previously (Abe et al., 2018). Anti-YAP, anti-histone H1, anti-CYP3A4 (Yoshinari et al., 2006), antiCYP2B1 (Yoshinari et al., 2006), anti-CYP1A2 (Yoshinari et al., 2006), and anti-calreticulin antibodies were used as primary antibodies for Western blotting.

Determination of P450 Activity. The CYP3A enzymatic activities in the microsomal fractions of mouse livers were determined using the P450-Glo CYP3A4 assay with Luciferin-IPA (Promega) according to the manufacturer's protocol.

TABLE 1

Primers and oligonucleotides for plasmid preparation

\begin{tabular}{lll}
\hline Plasmid Name or Mutated Position & Forward/Reverse & \multicolumn{1}{c}{ Sequence $\left(5^{\prime}\right.$ to $\left.3^{\prime}\right)$} \\
\hline mYAP-5SA-Flag-pTargeT (Flag-tagged) & Forward & TAGAATCTTTCCCGGGGGTACCGTCGACTG \\
& Reverse & CTTGTCATCGTCATCCTTGTAGTCTAACCACGTGAGAAAGCTTTC \\
hYAP-pTargeT & Forward & AGAAGCCATGGATCCCGGGCAGCAG \\
& Reverse & GCCTGAGGGCTCTATAACCATGTAAGAAAG \\
hYAP-S61A & Forward & CGCGGAGACCGACCTGGAGGCGCTCTTC \\
& Reverse & TCCCCGCGGACGTGCACGATCTG \\
hYAP-S109A & Forward & GCTACTGATGCAGGCACTGCAGGAGCCC \\
& Reverse & GGCCTGTCGGGAGTGGGATTTGGGC \\
hYAP-S127A & Forward & GCCTCTCCAGCTTCTCTGCAGTTGGGAGC \\
& Reverse & ATGAGCTCGAACATGCTGTGGAGTC \\
hYAP-S164A & Forward & GCTTTTGAGATACCTGATGATGTACCTC \\
& Reverse & AGACTGTCGAAGATGCTGAGCTGTGGGTG \\
hYAP-S397A & Forward & GCTACAGACAGTGGACTAAGCATGAGCA \\
& Reverse & CTCATCTCGAGAGTGATAGGTGCCAC \\
mPXR-V5-pTargeT (V5-tagged) & Forward & TCTCCTCGGTCTCGATTCTACGTGAGTGGCTGCCCCTGAAATCTTTCCCGGGGG \\
& Reverse & GGGTTAGGGATAGGCTTACCGCCATCTGTGCTGCTAAATAACTCTTGC \\
mYAP-WT and -5SA-pENTR & Forward & CACCATGGAGCCCGCGCAACAG \\
hYAP-WT and -5SA-pENTR & Reverse & CTATAACCACGTGAGAAAGCTT \\
& Forward & CACCATGGATCCCGGGCAGCAGCC \\
& Reverse & GCCTGAGGGCTCTATAACCATGTAAGAAAG \\
\hline
\end{tabular}


TABLE 2

Primers for quantitative reverse transcription PCR

\begin{tabular}{|c|c|c|}
\hline Gene & Forward Primer ( $5^{\prime}$ to $\left.3^{\prime}\right)$ & Reverse Primer ( $5^{\prime}$ to $\left.3^{\prime}\right)$ \\
\hline Ccna2 & GCAGCCTGCAAACTGTAAGGT & AATGACTCAGGCCAGCTCTGT \\
\hline Mcm2 & TCACGGTGCGCCACATCGAG & CCGGGCAAAAGTCTTGCGCA \\
\hline Ctgf & AAATGCTGCGAGGAGTGGGT & AAATGCTGCGAGGAGTGGGT \\
\hline Cyp2b10 & AAAGTCCCGTGGCAACTTCC & CATCCCAAAGTCTCTCATGG \\
\hline Сур3а11 & ACAAGCAGGGATGGACCTGG & TGTGACAGCAAGGAGAGGCG \\
\hline Myc & CACCAGCAGCGACTCTGA & GGGGTTTGCCTCTTCTCC \\
\hline Pxr & GGTGTGGTCCAGCGCAGCGT & ACTGCTGGGTTTGCTGGGCGT \\
\hline Rbl2 & TGCAGCCAGCTCGGAGGAAG & CATTTCCCTCCAGCGTGTAGCTCT \\
\hline Yap & TGCCCCAGACGCTGATGAAT & GGTTCATGGCAAAACGAGGGT \\
\hline $18 \mathrm{~S}$ rRNA & ACCGCGGTTCTATTTTGTTG & AGTCGGCATCGTTTATGGTC \\
\hline$A C T B$ & GCCAACACAGTGCTGTCTG & CCTGCTTGCTGATCCACATC \\
\hline ANKRD1 & AGCGCCCGAGATAAGTTGCT & CGATAAGATGCTCCGCGCAC \\
\hline$C A R$ & TCATCCATCACCAGCCCTTG & CGGGCTCCATCTTCAATTGTG \\
\hline CTGF & CCTGGTCCAGACCACAGAGT & TGGAGATTTTGGGAGTACGG \\
\hline CYP2B6 & CATCATCCCCAAGGACACAG & AAATCCGCTTCCCTAAGGAG \\
\hline CYP3A4 & CTGTGTGTTTCCAAGAGAAGTTAC & TGCATCAATTTCCTCCTGCAG \\
\hline CYR61 & ACCGCTCTGAAGGGGATCT & ACTGATGTTTACAGTTGGGCTG \\
\hline$G A P D H$ & CATGGGTGTGAACCATGAGAA & GGTCATGAGTCCTTCCACGAT \\
\hline$P X R$ & CAGATCTCCCTGCTGAAG & GAGAAGAGGGAGATGGCC \\
\hline YAP & GCAAGAACTGCTTCGGCAGG & CGCAGGGCTAACTCCTGACAT \\
\hline
\end{tabular}

rRNA, ribosomal RNA.

Statistical Analysis. Statistical analyses were performed using JMP Pro 12 (SAS Institute, Cary, NC). The significance of differences between the control and treated groups was assessed by Student's $t$ test or one-way ANOVA, followed by Dunnett's test or Tukey-Kramer test when data from two and multiple groups were compared, respectively.

\section{Results}

Influence of PXR Activation on YAP Nuclear Translocation in Mouse Livers. To investigate whether PXR activation could promote nuclear YAP accumulation in the liver, mice were treated intraperitoneally with vehicle, PCN (an mPXR ligand), or TCPOBOP (a mouse CAR ligand) for 3 days. PCN or TCPOBOP treatment increased liver-to-body weight ratios and induced centrilobular hepatocyte hypertrophy (Fig. 1, A and B). The number of Ki-67-positive nuclei and c-MYC (Myc) mRNA levels were remarkably increased in the livers of TCPOBOP-treated, but not PCN-treated, mice (Fig. 1, B and C), indicating that hepatocyte proliferation was selectively induced by CAR activation. The mRNA levels of Cyp3a11, a representative target gene of both PXR and CAR, were increased by both treatments and Cyp2b10 (a preferential target gene of CAR) mRNA levels were also remarkably increased following TCPOBOP administration (Fig. 1C). Under these conditions, in which both nuclear receptors were activated, nuclear YAP protein levels were markedly elevated (Fig. 1D).
A

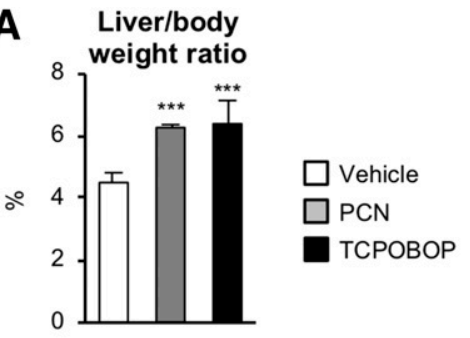

B
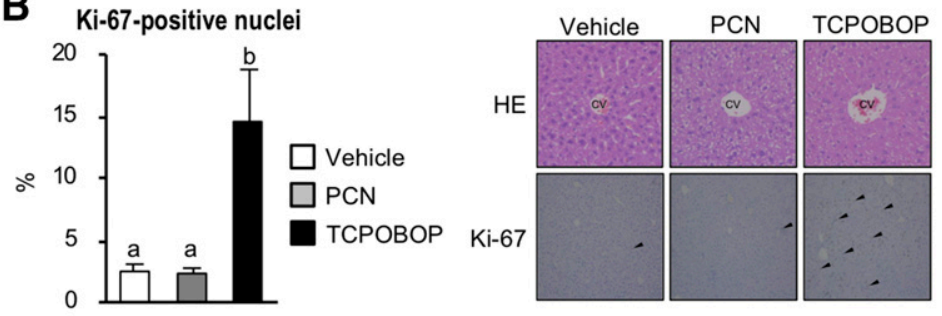

C

Cyp3a11
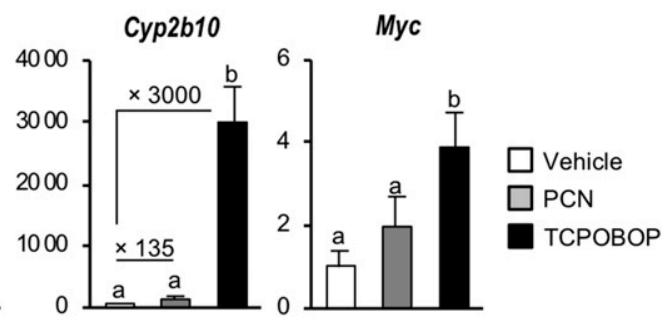

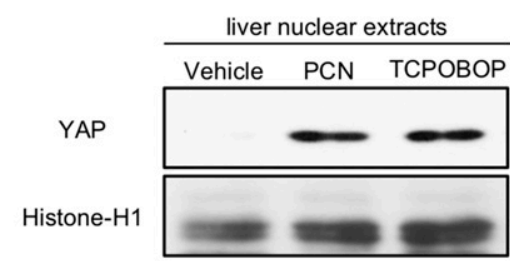

Fig. 1. Influence of nuclear receptor activation on nuclear YAP accumulation in mouse livers. C57BL/6 mice were treated with PCN (100 mg/kg), TCPOBOP (3 mg/kg), or vehicle (corn oil, $20 \mathrm{ml} / \mathrm{kg}$ ) once a day for three consecutive days. (A) Liver-to-body weight ratios were calculated. Values are the mean \pm S.D. $(n=4)$. $* * * P<0.001$ (vs. vehicle group; Dunnett's test). (B) Total and Ki-67-positive nuclei were counted in five randomly selected areas of each section (one section per mouse). Values are the mean \pm S.D. $(n=3)$. Columns not sharing a common letter $(\mathrm{a}$ and $\mathrm{b})$ differ significantly from each other $(P<0.05$; Tukey-Kramer test). (C) Hepatic total RNA was subjected to quantitative reverse transcription PCR. Relative mRNA levels were calculated by the $\Delta \Delta \mathrm{Ct}$ method using Actb mRNA as a reference. Values are the mean \pm S.D. $(n=4)$. Columns not sharing a common letter $(\mathrm{a}$, $\mathrm{b}$, and $\mathrm{c})$ differ significantly from each other $(P<0.05$; Tukey-Kramer test). (D) Nuclear extracts were subjected to western blot analysis with anti-YAP and antihistone-H1 antibodies. The images are from one representative experiment of two independent experiments. 
Next, we investigated chronological changes in liver hypertrophy and YAP activation in the livers of PCN- or TCPOBOPtreated mice after the time point when nuclear translocation of YAP had been observed in Fig. 1D. As shown in Fig. 2A, treatment with both activators increased liver-to-body weight ratios 24 hours after the treatment, as shown in Fig. 1, but differences in the timing of this effect were observed between treatments. While the liver-to-body weight ratio of TCPOBOP-treated mice continued to increase until at least 6 days after the last treatment, an increase in the liver-to-body weight ratio of PCN-treated mice was observed on only days 1 and 3 (Fig. 2A). This difference was also observed by histochemical analyses. In TCPOBOP-treated mice, centrilobular hepatocyte hypertrophy was observed even at day 9 , while it was observed at only day 1 but not day 9 in PCN-treated mice (Fig. 2B). Intriguingly, both PXR and CAR activation increased the mRNA levels of a YAP target gene, Ctgf. In PCNtreated mice, hepatic $C \operatorname{tg} f$ mRNA levels increased and peaked at day 6, while $C \operatorname{tg} f$ mRNA levels peaked in TCPOBOPtreated mice at day 9 (Fig. 2C). Treatment with TCPOBOP, but not PCN, increased mRNA levels of Birc5, another YAP target gene, and the cell proliferation marker genes Ccna2 and $M c m 2$, as expected (Fig. 2C). To evaluate PXR and CAR activation at these time points, we determined hepatic mRNA, protein, and enzymatic activity levels of CYP3As, which are induced by CAR and PXR activation. The levels of these parameters were significantly increased by both activators, and were sustained at high levels until 9 days after the last injection; however, the extent of the increase in CYP3As by PCN treatment was lower on days 6 and 9 (Fig. 2, C-E). The mRNA levels of Cyp2b10, a preferred CAR target gene, showed a pattern similar to those of Cyp3a11 (Fig. 2C). The protein levels of CYP2Bs remained increased after TCPOBOP or PCN treatment, although the increase in CYP2B levels following PCN treatment was lower at days 6 and 9 , as was the case for CYP3As (Fig. 2E). As a control, CYP1A protein levels were also determined and found to be increased by treatment with TCPOBOP but not PCN (Fig. 2E), as expected from our previous report showing CYP1A gene regulation by CAR (Yoshinari et al., 2010). Moreover, the protein levels of calreticulin, a representative microsomal protein, were not affected by either treatment at any time point (Fig. 2E).

These results suggest that PXR activation induces the nuclear accumulation of active YAP, as is the case for CAR. Furthermore, PXR-dependent liver hypertrophy might be caused by the induction of $\mathrm{P} 450$ and other factors, including YAP, because there was no clear correlation between the degree of P450 induction and hepatocyte hypertrophy after PCN treatment.

Influence of PXR Activation on YAP/TEAD-Dependent Gene Expression. Based on the results obtained in the in vivo experiments, we next investigated whether PXR activation could promote YAP/TEAD-dependent gene expression like CAR. YAP contains five serine residues that are phosphorylated by Hippo pathway kinases LATS1/2, which inactivate YAP. To clearly detect the effects of YAP/TEAD on gene transcription, we generated plasmids expressing the dominant active form of human or mouse YAP (hYAP-5SApTargeT and mYAP-5SA-pTargeT, respectively), in which all five phosphorylation sites were mutated to alanine (Zhao et al., 2007; Yabuta et al., 2013; Chiba et al., 2016; Abe et al., 2018). HepG2 cells were infected with WT hYAP- or dominant active hYAP-expressing adenovirus (Ad-hYAP-WT or AdhYAP-5SA, respectively), or control Ad-LacZ, and then quantitative reverse transcription PCR analyses were performed 48 hours after infection. As shown in Fig. 3A, YAP mRNA levels were increased in a multiplicity of infection (MOI)-dependent manner with both hYAP-expressing adenoviruses. In this model, the mRNA levels of the YAP target genes (CYR61, ANKRD1, and $C T G F)$ were also increased in an MOI-dependent manner following infection with Ad-hYAP5SA but not Ad-hYAP-WT (Fig. 3A). To determine whether PXR can promote YAP-dependent gene expression, HepG2 cells were coinfected with Ad-hYAP-5SA and hPXRexpressing adenovirus (Ad-hPXR) and treated with rifampicin, an hPXR activator. The Ad-hYAP-5SA-dependent gene expression of CYR61, ANKRD1, and CTGF was enhanced by Ad-hPXR coinfection with rifampicin, while hPXR activation alone did not significantly increase their expression levels (Fig. 3B). Furthermore, the mRNA levels of $P X R$ and its target gene, CYP3A4, were increased by Ad-hPXR infection and clearly decreased by coinfection with Ad-hYAP-5SA. Unexpectedly, hPXR expression increased the endogenous (Fig. 3B, lane 1 vs. 2) and adenovirus-induced expression (Fig. 3B, lane 3 vs. 4) of $Y A P$, and hYAP-5SA expression tended to decrease the endogenous (Fig. 3B, lane 1 vs. 3) and significantly decreased adenovirus-induced expression of PXR (Fig. 3B, lane 2 vs. 4), suggesting the presence of a post-transcriptional interaction between YAP and PXR.

To investigate whether activated PXR has any influence on YAP/TEAD-mediated gene transcription, we performed reporter assays using TEAD-responsive reporter plasmid (TEAD-pTA). In COS-1 cells, the reporter activities were increased by hYAP-5SA expression, and hPXR activation enhanced this increase in a dose-dependent manner, whereas hPXR activation alone did not affect reporter activity (Fig. 3C). This enhancement in reporter activity was also observed with the combined expression of mYAP-5SA and mPXR (Fig. 3D).

Influence of YAP Overexpression on PXR-Dependent Gene Expression. We next investigated whether YAP could affect PXR-mediated gene expression. In mouse primary hepatocytes, PCN treatment increased Cyp $3 a 11$ mRNA levels and the increases were remarkably suppressed by mYAP-WT or mYAP-5SA overexpression (Fig. 4A).

To assess the species difference in PXR-dependent gene expression between mice and humans, similar assays were carried out with HepG2 cells. In control adenovirus-infected HepG2 cells, the rifampicin-dependent expression of CYP3A4 was observed but drastically suppressed or showed a tendency to decrease following Ad-hYAP-5SA and Ad-hYAP-WT infection, respectively (Fig. 4B), indicating no clear difference in this phenomenon between species. The basal CYP3A4 and PXR mRNA levels also showed a tendency to decrease with hYAP-5SA overexpression, as shown in Fig. 3B (Fig. 4B).

To confirm the YAP-induced suppression of PXR-dependent gene transcription, reporter assays were conducted with a reporter plasmid containing three PXR-responsive elements (p3A4) (Toriyabe et al., 2009). As expected, the increase in mPXR/PCN-dependent luciferase activity was suppressed by mYAP expression, and this suppression was more pronounced following mYAP-5SA expression rather than mYAP-WT (Fig. 4C). These suppressing effects of YAP were also observed in a human cell model (Fig. 4D). Under these experimental 
A

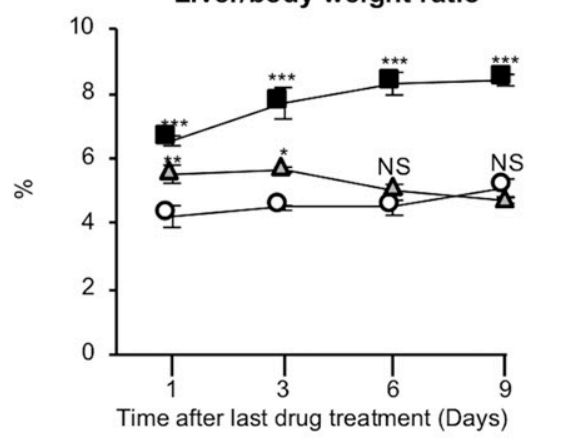

C

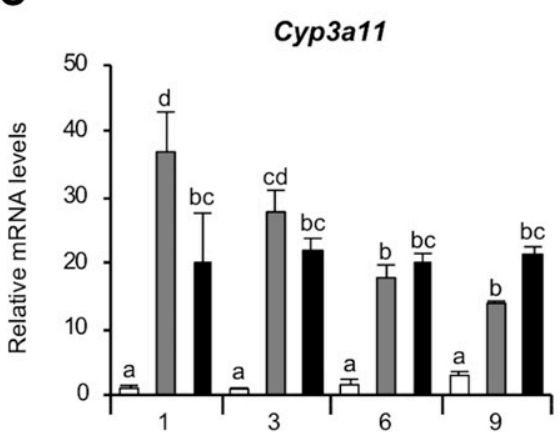

Time after last drug treatment (Days)

Cyp2b10

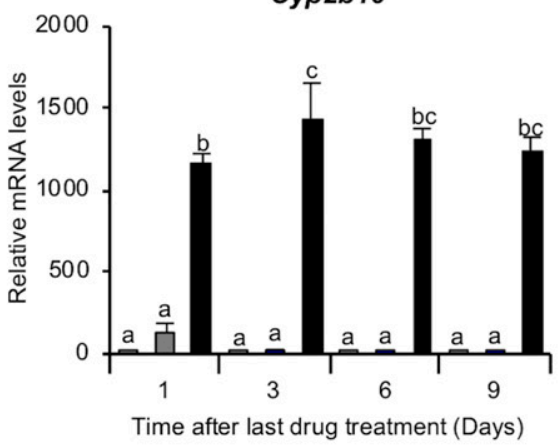

D

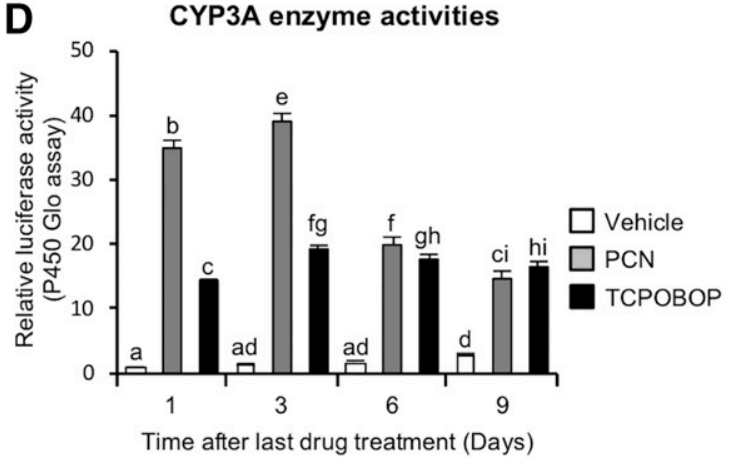

B
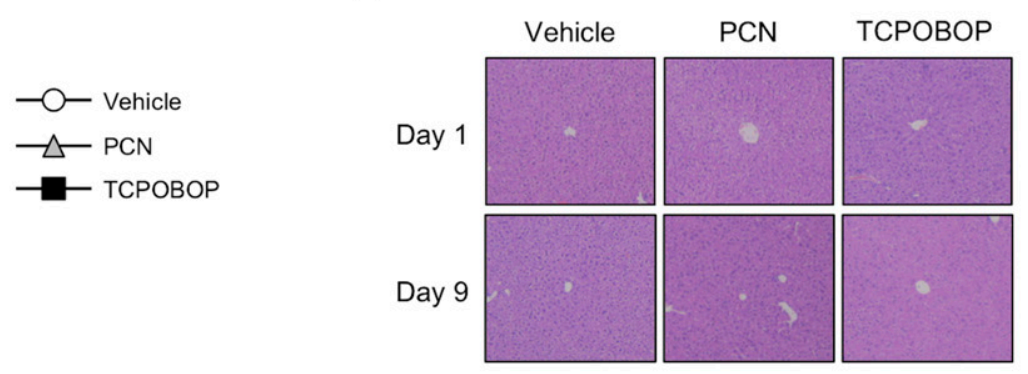
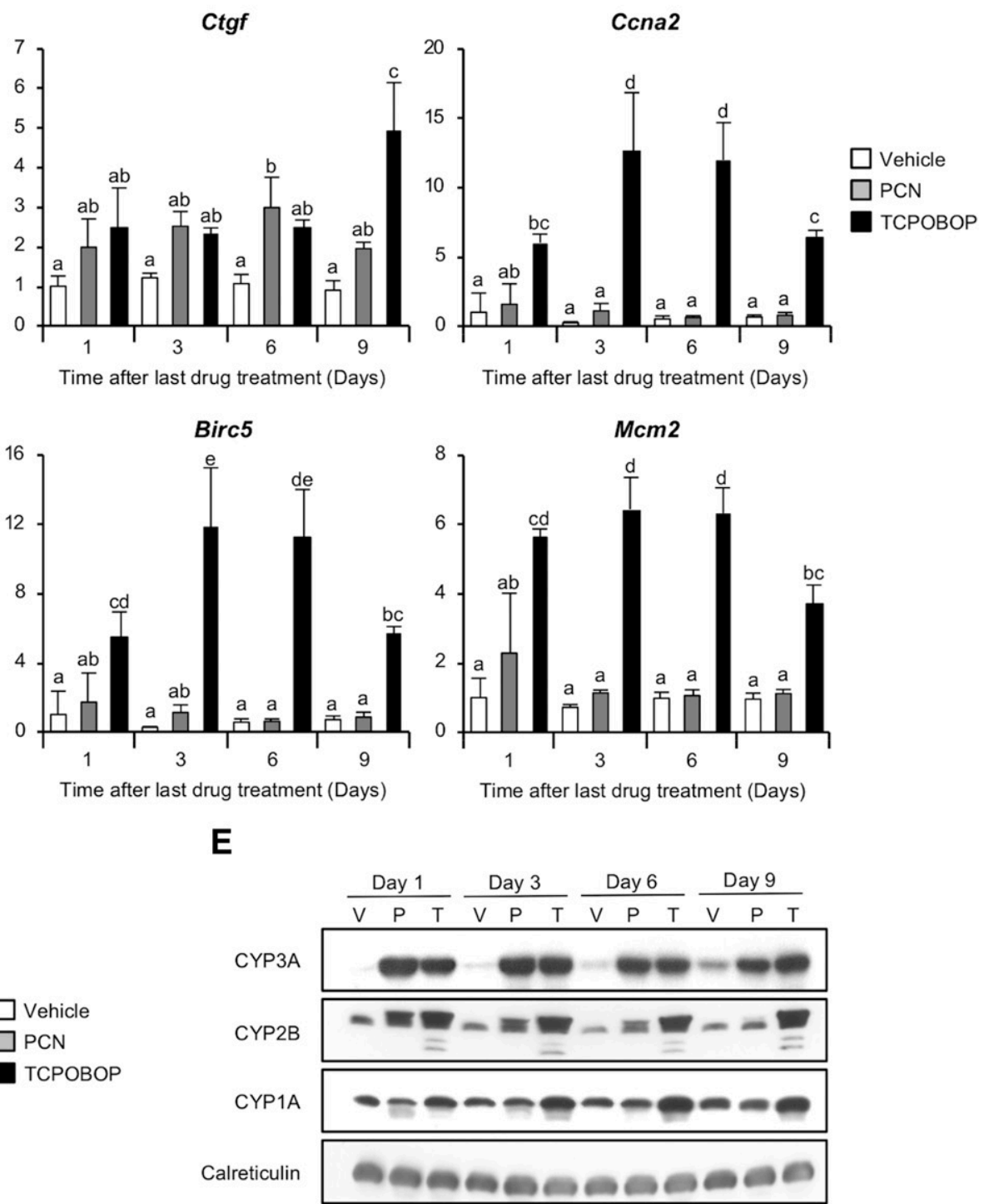

Fig. 2. Chronological analysis of nuclear receptor activation in mouse livers. C57BL/6 mice were treated with PCN (100 mg/kg), TCPOBOP $(3 \mathrm{mg} / \mathrm{kg})$, or vehicle (corn oil, $20 \mathrm{ml} / \mathrm{kg}$ ) once a day for 3 days, and sacrificed at 1, 3, 6, or 9 days after the last treatment. (A) Liver-to-body weight ratios were calculated. Values are the mean \pm S.D. $(n=3) . * P<0.05 ; * * P<0.01 ; * * P<0.001$, NS, not significant (Dunnett's test vs. vehicle treatment). (B) Livers were fixed and stained with hematoxylin and eosin. (C) Hepatic total RNA was subjected to quantitative reverse transcription PCR. Relative mRNA levels were calculated by the $\Delta \Delta \mathrm{Ct}$ method using $18 \mathrm{~S}$ ribosomal RNA as a reference. Values are the mean \pm S.D. $(n=3)$. Columns not sharing a common letter ( $\mathrm{a}, \mathrm{b}, \mathrm{c}, \mathrm{d}$, and e) differ significantly from each other $(P<0.05$; Tukey-Kramer test). (D) CYP3A enzyme activities of each liver microsomal fraction were measured by using the P450-Glo assay system. Values are the mean \pm S.D. $(n=3)$. Columns not sharing a common letter $(a, b, c, d, e, f, g, h$, and i) differ significantly from each other $(P<0.05$; Tukey-Kramer test). (E) Liver microsomal fractions were subjected to western blot analysis with antibodies against CYP3A4, CYP2B1, CYP1A2, or calreticulin. 
A

YAP

CYR61

ANKRD1

CTGF

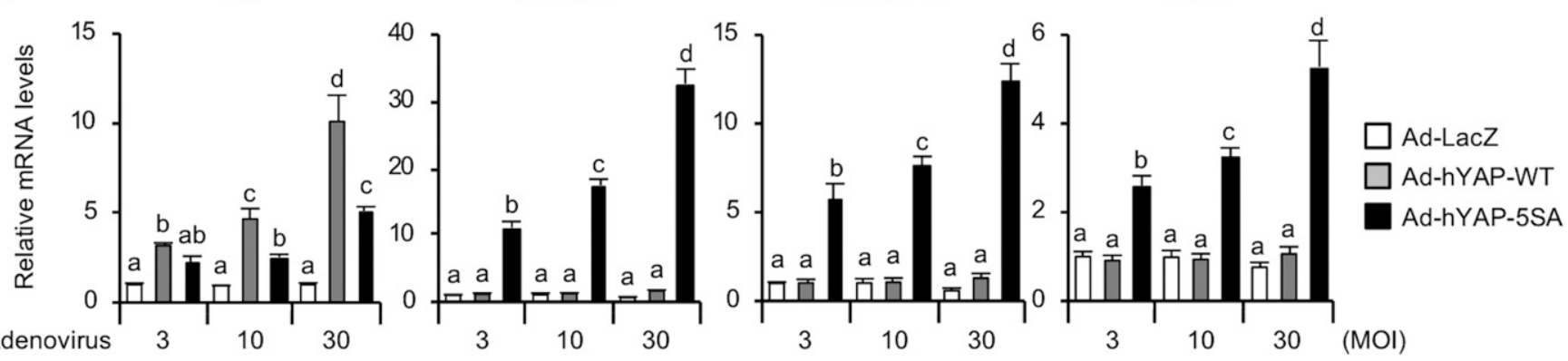

B
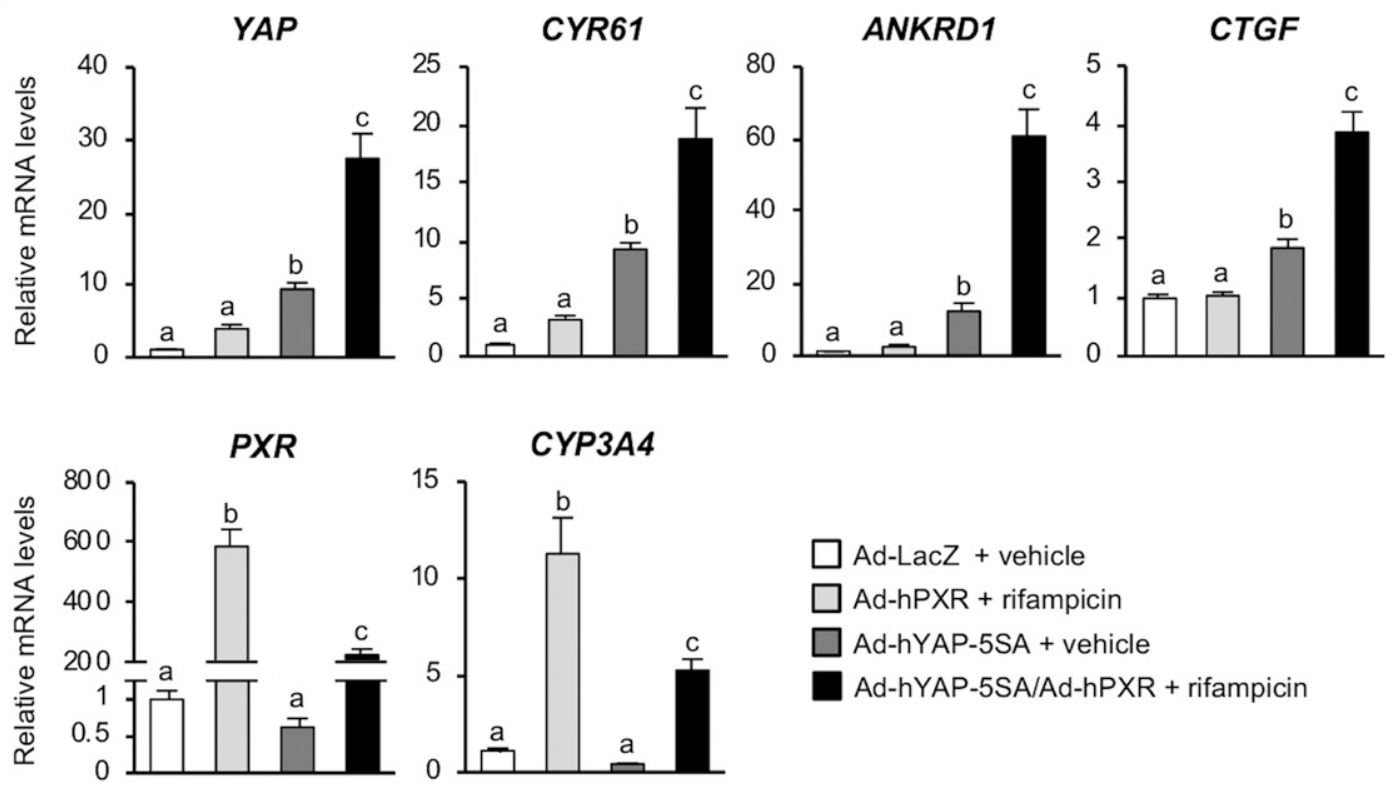

C

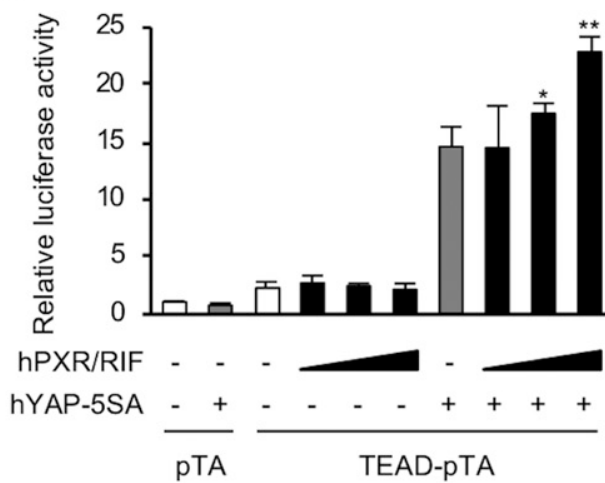

D

Fig. 3. Influence of PXR activation on YAP-dependent gene expression. HepG2 cells were precultured for 48 hours and then infected with each adenovirus for an additional 48 hours in (A and B). In (B), 48 hours after adenovirus infection at an MOI of 10, the cells were treated with vehicle $(0.1 \%$ DMSO) or $10 \mu \mathrm{M}$ rifampicin for 24 hours. Total RNA was subjected to quantitative reverse transcription PCR. Relative mRNA levels were calculated by the $\Delta \Delta \mathrm{Ct}$ method using $18 \mathrm{~S}$ ribosomal RNA as a reference. Columns not sharing a common letter (a, b, and c) differ significantly from each other $(P<0.05$; Tukey-Kramer test). (C) COS-1 cells were transfected with the reporter plasmid (TEAD-pTA or pTA: $100 \mathrm{ng})$, phRL-TK (10 ng), and expression plasmid (hYAP-5SA: $50 \mathrm{ng}$; hPXR: $0.1,1 \mathrm{or} 10 \mathrm{ng}$ ) for 24 hours and then treated with vehicle $(0.1 \%$ DMSO) or $10 \mu \mathrm{M}$ rifampicin for additional 24 hours in serum-free medium. (D) COS-1 cells were transfected with the reporter plasmid (TEAD-pTA or pTA: $100 \mathrm{ng}$ ), phRL-TK (10 ng), and expression plasmid (mYAP-5SA: $50 \mathrm{ng}$; mPXR: 0.1, 1 or $10 \mathrm{ng}$ ) for 24 hours and then treated with vehicle $(0.1 \%$ DMSO) or $10 \mu \mathrm{M}$ PCN for an additional 24 hours in serum-free medium. Reporter activities were determined with the Dual-Glo Luciferase Assay System. Values are the mean \pm S.D. $(n=4)$. ${ }^{*} P<0.05 ; * * P<0.01$ (Dunnett's test vs. cells expressing TEAD-pTA and YAP-5SA alone). All the values in (B-D) are the mean \pm S.D. of quadruplicate samples in a representative experiment among at least two independent experiments.

conditions, mYAP-WT or mYAP-5SA expression did not decrease Pxr mRNA levels (data not shown).

Furthermore, the influence of YAP knockdown on hPXR-mediated CYP3A4 expression was investigated in
HepG2 cells (Fig. 4E). The rifampicin-mediated CYP3A4 induction was greater in the $Y A P$-knockdown cells, where YAP mRNA levels were reduced by $\sim 95 \%$, than in control siRNA-transfected cells. These results suggest that YAP 
A

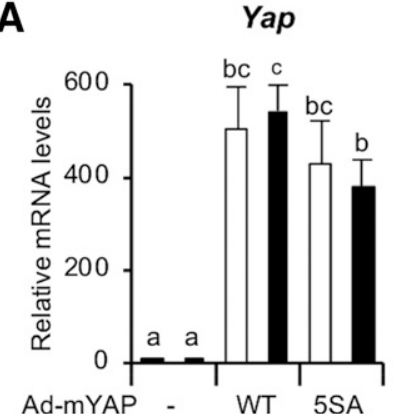

B

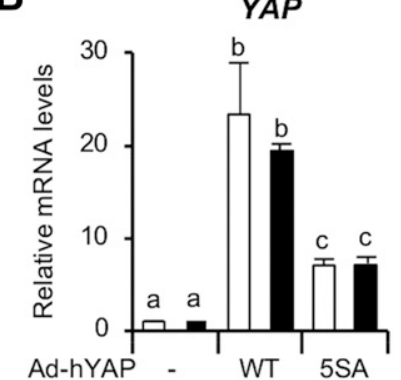

C

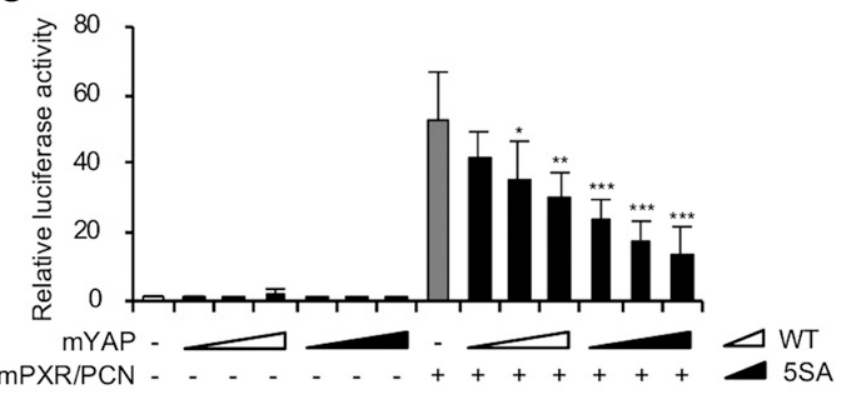

CYP3A4

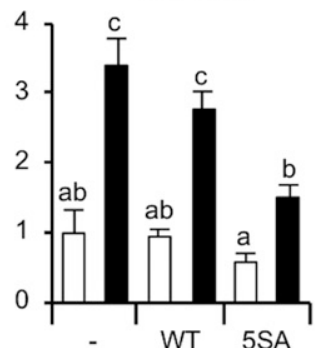

Pxr

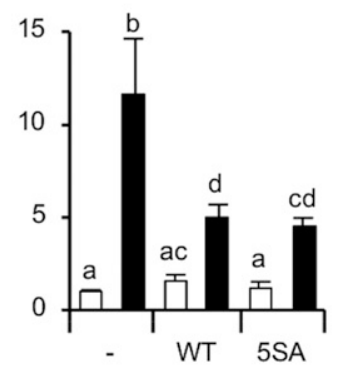

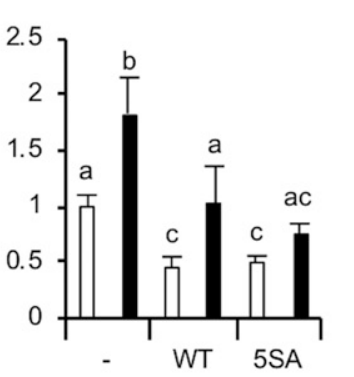

Vehicle

PCN
E

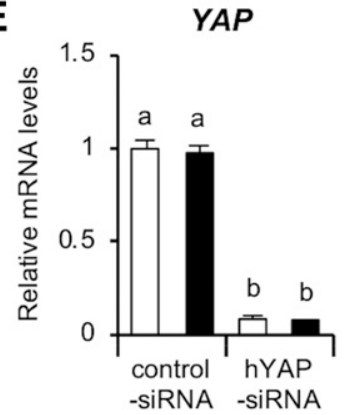

PXR

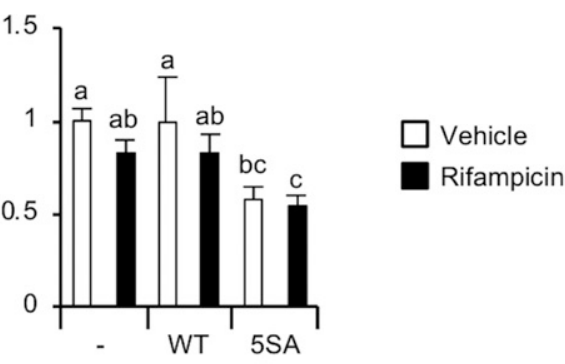

D

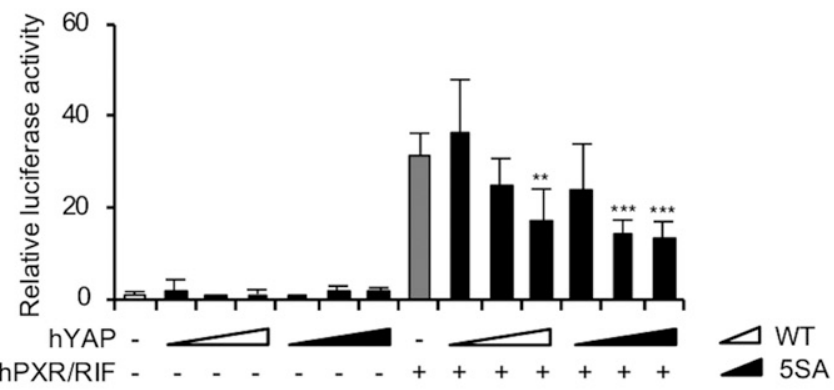

Fig. 4. Influence of YAP overexpression on PXR-mediated gene expression. (A) Isolated mouse primary hepatocytes were seeded in collagen type I-coated 24-well plates. Four hours later, the cells were infected with Ad-LacZ (-), Ad-mYAP-WT (WT) or Ad-mYAP-5SA (5SA) at an MOI of 10 for 48 hours and then treated with vehicle $(0.1 \% \mathrm{DMSO}$ ) or $10 \mu \mathrm{M}$ PCN for 24 hours. Total RNA was subjected to quantitative reverse transcription PCR (qRTPCR). Relative mRNA levels were calculated by the $\Delta \Delta$ Ct method using 18 S ribosomal RNA (rRNA) as a reference. Columns not sharing a common letter (a, b, c, and d) differ significantly from each other $(P<0.05$; Tukey-Kramer test). (B) HepG2 cells were precultured for 48 hours and then infected with each adenovirus at an MOI of 10 . Forty-eight hours later, the cells were treated with vehicle (0.1\% DMSO) or $10 \mu \mathrm{M}$ rifampicin for 24 hours. Total RNA was subjected to qRT-PCR. Relative mRNA levels were calculated by the $\Delta \Delta \mathrm{Ct}$ method using $18 \mathrm{~S}$ rRNA as a reference. Columns not sharing a common letter $(\mathrm{a}, \mathrm{b}$, and $\mathrm{c})$ differ significantly from each other $(P<0.05$; Tukey-Kramer test). (C) COS-1 cells were transfected with p3A4 (10 ng), phRL-TK $(57$ ng), and expression plasmid (mock or hPXR: $1 \mathrm{ng}$; hYAP-WT or hYAP-5SA: 1, 3, or $10 \mathrm{ng}$ ) for 24 hours and then treated with vehicle (0.1\% DMSO) or $10 \mu \mathrm{M}$ rifampicin for an additional 24 hours. (D) COS-1 cells were transfected with p3A4 (10 ng), phRL-TK (60 ng), and expression plasmid (mock or mPXR: $3 \mathrm{ng}$; mYAP-WT or mYAP-5SA: 1,10 or $30 \mathrm{ng})$ for 24 hours and then treated with vehicle (0.1\% DMSO) or $10 \mu \mathrm{M}$ PCN for an additional 24 hours. Reporter activities were determined with Dual-Glo Luciferase Assay Systems. ${ }^{*} P<0.05$; ${ }^{* *} P<0.01$; ${ }^{* * *} P<0.001$ (Dunnett's test vs. each of hPXR/ rifampicin or mPXR/PCN alone). (E) HepG2 cells were reverse transfected with $10 \mathrm{nM}$ control-siRNA or hYAP-siRNA. Forty-eight hours later, the cells were treated with vehicle ( $0.1 \%$ DMSO) or $10 \mu \mathrm{M}$ rifampicin for 24 hours. Total RNA was subjected to qRT-PCR. Relative mRNA levels were calculated by the $\triangle \triangle \mathrm{Ct}$ method using GAPDH mRNA as a reference. Columns not sharing a common letter $(\mathrm{a}, \mathrm{b}$, and $\mathrm{c})$ differ significantly from each other $(P<0.05$; Tukey-Kramer test). All of the values in (A-E) are the mean \pm S.D. of quadruplicate samples in a representative experiment among at least two independent experiments. 
acts as a corepressor in PXR-dependent gene transcription.

Roles of YAP in the PXR-Mediated Enhancement of Hepatocyte Proliferation. Recently, we reported that activated mPXR enhances hepatocyte proliferation induced by various types of growth stimuli in vivo and in vitro through the inhibition of FOXO3-dependent gene transcription, although mPXR activation alone does not induce hepatocyte proliferation (Shizu et al., 2013). For a more detailed understanding of the relationship between YAP and PXR, we investigated whether YAP could play a role in the PXRmediated increase in cell proliferation in immortalized murine hepatocyte AML12 cells.

In AML12 cells under serum-free conditions, the cell cycle was completely arrested, and we were able to induce cell growth by treatment with medium containing $10 \%$ serum (Shizu et al., 2016). In AML12 cells, mYAP-siRNA transfection reduced Yap mRNA levels by $\sim 70 \%$ (Fig. 5A). As shown in Fig. 5B, serum-induced cell proliferation was enhanced by mPXR activation in the control cells, but this enhancement was not observed in Yap-knockdown cells. As shown by quantitative reverse transcription PCR, the mRNA levels of $R b l 2$, a FOXO3 target gene that encodes a cell cycle suppressor, were decreased by mPXR activation, as expected from our previous findings (Shizu et al., 2013), but this decrease disappeared completely following Yap knockdown (Fig. 5C). In addition, both the basal and PXR-induced expressions of Cyp3a11 were increased by Yap knockdown (Fig. 5C), which is consistent with the results shown in Fig. 4.

YAP-Dependent Regulation of Liver Functions Related to Drug Metabolism. We finally investigated the influence of YAP overexpression on hepatic functions in HepaRG cells, which are primary human hepatocyte-like cells that express high levels of P450s and liver-enriched transcription factors such as $\mathrm{HNF} 4 \alpha$, PXR, and CAR. In HepaRG cells infected with Ad-hYAP-5SA, the rifampicininduced CYP3A4 mRNA levels and rifampicin- or CITCO, a human CAR ligand-induced CYP2B 6 mRNA levels were significantly lower than those in control virus-infected cells. The basal expression levels of CYP3A4 and $C Y P 2 B 6$ were also significantly, and in an MOI-dependent manner, reduced by hYAP-5SA expression. Consistently, $H N F 4 \alpha, P X R$, and $C A R$ mRNA levels were also remarkably decreased by Ad-hYAP5SA infection in an MOI-dependent manner (Fig. 6).

\section{Discussion}

We recently reported that $\mathrm{CAR}$ activation induces nuclear YAP accumulation and YAP/TEAD-dependent gene expression in mouse livers. In contrast, the relationship between PXR and YAP remains unclear. We thus investigated the functional interaction between PXR and YAP in liver hypertrophy and drug metabolism.

We first asked whether activated PXR can induce nuclear YAP accumulation and YAP-dependent gene expression in mouse livers. In PCN-treated mice, liver enlargement and hepatocyte hypertrophy were accompanied by increased YAP activation (Figs. 1 and 2), although YAP activation was more prominent and persistent in TCPOBOP-treated mice than in PCN-treated mice (Fig. 2C). In addition, while PXR was continuously active for 9 days after the last drug treatment, as judged by its target gene expression, the clear increases in YAP target gene expression, hepatocyte hypertrophy, and liver enlargement were not observed at that time point in PCN-treated mice. In contrast, these effects were observed at even 9 days after the last drug treatment in TCPOBOPtreated mice. These results indicate that PXR, like CAR,
A
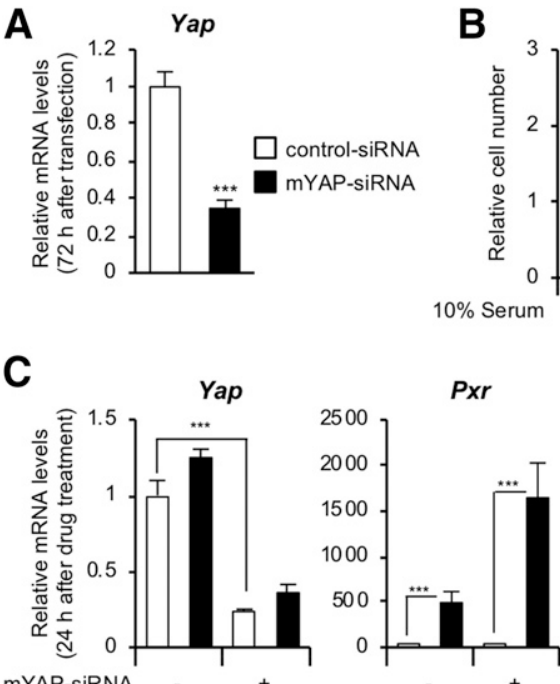

mYAP-SIRNA
B

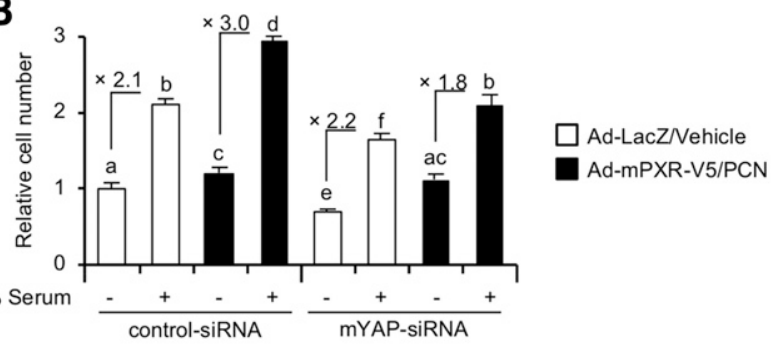

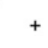

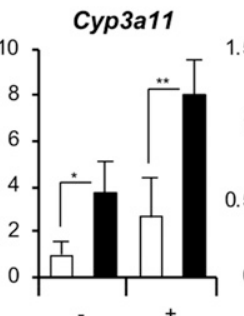

$R b / 2$

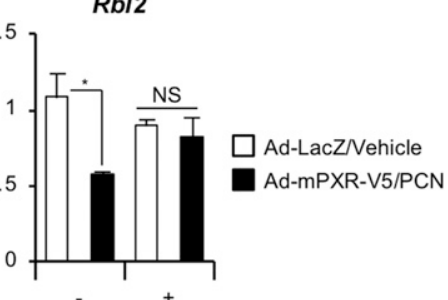

Fig. 5. The role of YAP in the PXRmediated enhancement of cell proliferation. AML12 cells were transfected with $10 \mathrm{nM}$ control-siRNA or mYAP-siRNA for 24 hours and cultured in fresh serum-free medium for 48 hours. Twenty-four hours after siRNA transfection, the cells were infected with Ad-LacZ or Ad-mPXR-V5 at an MOI of 30 for 48 hours in serum-free medium. Forty-eight hours after infection, the cells were treated with vehicle $(0.1 \% \mathrm{DMSO})$ or $10 \mu \mathrm{M} \mathrm{PCN}$ in the presence or absence of $10 \%$ serum. (A) Total RNA was extracted 72 hours after siRNA transfection and subjected to quantitative reverse transcription PCR (qRT-PCR). ${ }^{* * *} P \leq 0.001$ (vs. controlsiRNA group; Student's $t$ test). (B) The cell numbers were calculated using the WST-8 assay 48 hours after serum treatment. Columns not sharing a common letter ( $a, b, c, d, e$, and f) differ significantly from each other $(P<0.05$; TukeyKramer test). (C) Total RNA was extracted 24 hours after drug treatment and subjected to qRT-PCR. Relative mRNA levels were calculated by the $\Delta \Delta \mathrm{Ct}$ method using $A c t b$ as a reference. ${ }^{*} P<$ $0.05 ; * * P<0.01 ; * * * P<0.001$ (Student's $t$ test). All of the values in $\mathrm{A}-\mathrm{C}$ are the mean \pm S.D. of quadruplicate samples in a representative experiment among at least two independent experiments. 
YAP
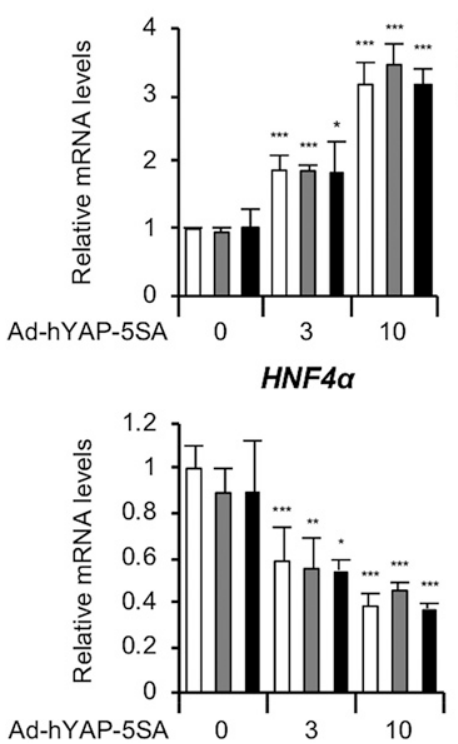

CYP3A4
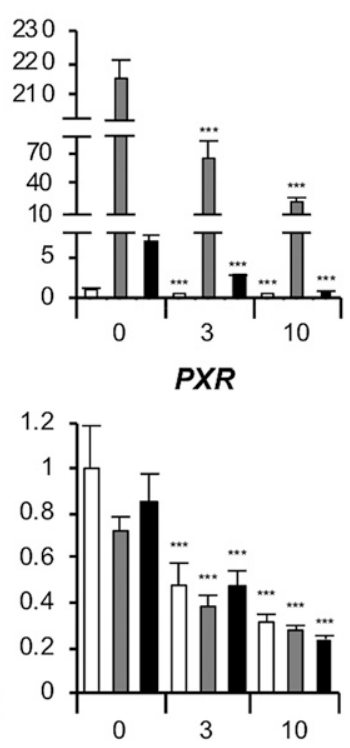

CYP2B6
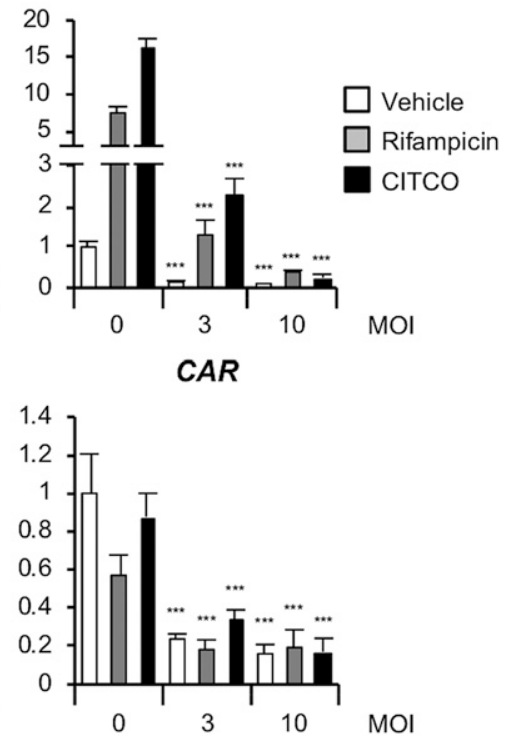

Fig. 6. Influence of YAP activation on the expression levels of genes associated with liver function in HepaRG cells. HepaRG cells were seeded in collagen type I-coated 48-well plates and cultured in HepaRG working medium. Six hours later, the medium was replaced with fresh working medium, and the cells were cultured for an additional 66 hours. The cells were infected with Ad-LacZ or Ad-hYAP5SA (MOI of 0,3 , or 10 ) for 48 hours and then cultured in serum-free induction medium containing each drug for an additional 48 hours. The drug concentrations used were as follows: vehicle (0.1\% DMSO), $10 \mu \mathrm{M}$ rifampicin, and $0.5 \mu \mathrm{M}$ CITCO. Total RNA was subjected to quantitative reverse transcription PCR. Relative mRNA levels were calculated by the $\Delta \Delta \mathrm{Ct}$ method using $18 \mathrm{~S}$ ribosomal RNA as a reference. Values are the mean \pm S.D. $(n=4) .{ }^{*} P<0.05 ; * P<0.01$; $* * * P<0.001$ (comparison between the 0 MOI groups for each drug; Dunnet's multiple comparisons test). The experiments were performed independently at least two times. promotes YAP activation, but the activation potency of YAP is relatively low compared with that of CAR.

These results also suggest a possible feedback mechanism that prevents the sustained interaction between PXR and YAP (i.e., negative feedback). PXR activation enhanced YAP/ TEAD-dependent gene transcription, while YAP expression suppressed PXR-dependent gene transcription in human and mouse models (Figs. 3 and 4). We previously demonstrated a functional interaction between CAR and YAP that promotes mutual activation of their target gene expression (i.e., positive feedback) (Abe et al., 2018). These differences between the mechanism by which PXR and CAR functionally interact with YAP might help clarify the differences in carcinogenic effects of both receptors and the mechanism of xenobiotic-dependent liver and hepatocyte hypertrophy.

In addition to crosstalk at the transcriptional level, in which PXR enhances YAP/TEAD-mediated gene transcription and YAP suppresses PXR-mediated gene transcription (Figs. 3C and 4, A and C), the present results also suggest an interaction between PXR and YAP at the post-transcriptional level. PXR overexpression increased endogenous YAP mRNA levels in HepG2 cells (Fig. 3B), and YAP overexpression reduced endogenous PXR mRNA levels in primary mouse hepatocytes, HepG2 cells, and HepaRG cells (Figs. 4, A and B, and 6). Interestingly and unexpectedly, these interactions were observed with exogenously expressed human PXR and YAP (Fig. 3B). At this moment, we do not know the mechanism of this phenomenon. Expressed YAP or PXR might act as a transcription factor to influence the stability or degradation of PXR and YAP mRNA, respectively, through an unknown mechanism, although we are unable to rule out the possibility that competition for the transcriptional machinery affects the efficacy of PXR and YAP gene expression.

PXR or CAR activation is known to induce centrilobular hypertrophy (Maronpot et al., 2010; Hall et al., 2012). Because of the preferential and abundant expression of $\mathrm{P} 450 \mathrm{~s}$ around the central vein, $\mathrm{P} 450$ induction is considered as a major cause of hepatocyte hypertrophy in this area (Maronpot et al., 2010; Hall et al., 2012). However, although both PXR-dependent P450 induction and hepatocyte hypertrophy were observed at several time points after PCN treatment, there was no clear correlation between the degree of $\mathrm{P} 450$ induction and hepatocyte hypertrophy in the mice at later time points (Fig. 2). For example, on day 9 , no hepatocyte hypertrophy was observed in PCN-treated mice but CYP3A protein and Cyp3a11 mRNA levels steadily increased. These results suggest that PXR activation-induced hepatocyte hypertrophy is mediated by not only P450 induction but also other factors, such as YAP and the Hippo pathway.

The causal relationship between YAP activation and cell hypertrophy has recently become apparent. YAP/TEAD activation promoted the expression of microRNA-29, which acted as a translational suppressor of PTEN and could induce cell hypertrophy through the microRNA-29-PTEN-AKT-mTOR pathway (Csibi and Blenis, 2012; Tumaneng et al., 2012). Autophagy was inhibited by the mTOR signaling pathway, and deficient liver-specific autophagy induced the hypertrophy of liver parenchymal cells (Komatsu et al., 2005; Jung et al., 2010). Based on these findings, hepatocyte hypertrophy induced by the activation of PXR and/or CAR might occur through multiple cellular events, including the suppression of autophagy associated with mTOR pathway activation, which is triggered by YAP activation. It would thus be of great interest to clarify the relationship between these nuclear receptors and the mTOR/autophagy pathway in a future study.

P450 expression is suppressed in liver tumors and hepatoma cell lines (Rodríguez-Antona et al., 2002), but the molecular basis of this suppression remains unclear. Since YAP activation is strongly associated with liver tumor formation, YAP activation might affect P450 expression. In this study, YAP overexpression reduced CYP3A4 and Cyp3a11 mRNA levels, while YAP knockdown increased these levels (Figs. 3C, 4, A, B, and E, and 5C). In addition, YAP suppressed 
PXR-mediated CYP3A4 expression (Fig. 4, C and D), and YAP overexpression reduced the expression of liver functionrelated genes (Fig. 6). Abnormally activated YAP reduced the expression of HNF4 $\alpha$ and FOXA2 upon the induction of liver or hepatocyte differentiation (Alder et al., 2014; Lee et al., 2016). In MST1 and MST2 null mice, YAP is abnormally activated, which results in deteriorated liver function and liver carcinoma formation; however, liver-specific Yap knockdown was able to restore liver morphology and functions comparable to those of normal mouse livers (Fitamant et al., 2015). The present results and our current knowledge imply that YAP modulates liver functions and homeostasis through inhibiting the expression and function of the liver-enriched nuclear receptors $\mathrm{HNF} 4 \alpha$, PXR, and CAR.

In summary, we have demonstrated functional crosstalk between PXR and YAP in xenobiotic-induced liver hypertrophy and drug metabolism. Over the course of our experiments, Dr. Bi's group reported the association between PXR and YAP in the regulation of liver size with multiple in vivo experiments (Jiang et al., 2019), which are consistent with the present results. In addition, we revealed that there is no difference in this crosstalk between humans and mice, while we found notable differences between PXR and CAR in terms of their interactions with YAP. Differences between the interactions of PXR and CAR with YAP may provide a new insight into understanding the mechanism of xenobiotic-induced liver hypertrophy and hepatocarcinogenesis.

\section{Authorship Contributions}

Participated in research design: Abe, Shizu, Sasaki, Hosaka, Kodama, Matsuzawa, Yoshinari.

Conducted experiments: Abe, Shizu, Sasaki, Shimizu, Yoshinari.

Performed data analysis: Abe, Shizu, Sasaki, Shimizu, Yoshinari.

Wrote or contributed to the writing of the manuscript: Abe, Shizu, Sasaki, Hosaka, Kodama, Matsuzawa, Yoshinari.

\section{References}

Abe T, Amaike Y, Shizu R, Takahashi M, Kano M, Hosaka T, Sasaki T, Kodama S, Matsuzawa A, and Yoshinari K (2018) Role of YAP activation in nuclear receptor CAR-mediated proliferation of mouse hepatocytes. Toxicol Sci 165:408-419.

Abe T, Takahashi M, Kano M, Amaike Y, Ishii C, Maeda K, Kudoh Y, Morishita T, Hosaka T, Sasaki T, et al. (2017) Activation of nuclear receptor CAR by an environmental pollutant perfluorooctanoic acid. Arch Toxicol 91:2365-2374.

Alder O, Cullum R, Lee S, Kan AC, Wei W, Yi Y, Garside VC, Bilenky M, Griffith M, Morrissy AS, et al. (2014) Hippo signaling influences HNF4A and FOXA2 enhancer switching during hepatocyte differentiation. Cell Rep 9:261-271.

Blanco-Bose WE, Murphy MJ, Ehninger A, Offner S, Dubey C, Huang W, Moore DD, and Trumpp A (2008) C-Myc and its target FoxM1 are critical downstream effectors of constitutive androstane receptor (CAR) mediated direct liver hyperplasia. Hepatology 48:1302-1311.

Butler WH (1978) Long-term effects of phenobarbitone-Na on male Fischer rats. $\mathrm{Br}$ $J$ Cancer 37:418-423.

Chiba T, Ishihara E, Miyamura N, Narumi R, Kajita M, Fujita Y, Suzuki A, Ogawa Y, and Nishina H (2016) MDCK cells expressing constitutively active Yes-associated protein (YAP) undergo apical extrusion depending on neighboring cell status. Sci Rep 6:28383.

Csibi A and Blenis J (2012) Hippo-YAP and mTOR pathways collaborate to regulate organ size. Nat Cell Biol 14:1244-1245.

Fitamant J, Kottakis F, Benhamouche S, Tian HS, Chuvin N, Parachoniak CA, Nagle JM, Perera RM, Lapouge M, Deshpande V, et al. (2015) YAP inhibition restores hepatocyte differentiation in advanced HCC, leading to tumor regression. Cell Rep 10:1692-1707.

Guo L and Teng L (2015) YAP/TAZ for cancer therapy: opportunities and challenges (review). Int J Oncol 46:1444-1452.

Hall AP, Elcombe CR, Foster JR, Harada T, Kaufmann W, Knippel A, Küttler K, Malarkey DE, Maronpot RR, Nishikawa A, et al. (2012) Liver hypertrophy: a review of adaptive (adverse and non-adverse) changes-conclusions from the 3rd International ESTP Expert Workshop. Toxicol Pathol 40:971-994.

Harvey KF, Zhang X, and Thomas DM (2013) The Hippo pathway and human cancer Nat Rev Cancer 13:246-257.

Hernandez JP, Mota LC, and Baldwin WS (2009) Activation of CAR and PXR by dietary, environmental and occupational chemicals alters drug metabolism, intermediary metabolism, and cell proliferation. Curr Pharmacogenomics Person Med 7:81-105.

Jiang Y, Feng D, Ma X, Fan S, Gao Y, Fu K, Wang Y, Sun J, Yao X, Liu C, et al. (2019) Pregnane $\mathrm{X}$ receptor regulates liver size and liver cell fate by yes-associated protein activation in mice. Hepatology 69:343-358.

Jung CH, Ro S-H, Cao J, Otto NM, and Kim D-H (2010) mTOR regulation of autophagy. FEBS Lett 584:1287-1295.

Kawamoto T, Kakizaki S, Yoshinari K, and Negishi M (2000) Estrogen activation of the nuclear orphan receptor CAR (constitutive active receptor) in induction of the mouse Cyp2b10 gene. Mol Endocrinol 14:1897-1905.

Kliewer SA, Goodwin B, and Willson TM (2002) The nuclear pregnane X receptor: a key regulator of xenobiotic metabolism. Endocr Rev 23:687-702.

Kodama S, Koike C, Negishi M, and Yamamoto Y (2004) Nuclear receptors CAR and PXR cross talk with FOXO1 to regulate genes that encode drug-metabolizing and gluconeogenic enzymes. Mol Cell Biol 24:7931-7940.

Komatsu M, Waguri S, Ueno T, Iwata J, Murata S, Tanida I, Ezaki J, Mizushima N, Ohsumi Y, Uchiyama Y, et al. (2005) Impairment of starvation-induced and constitutive autophagy in Atg7-deficient mice. J Cell Biol 169:425-434.

Lee CH, Inoki K, and Guan KL (2007) mTOR pathway as a target in tissue hypertrophy. Annu Rev Pharmacol Toxicol 47:443-467.

Lee DH, Park JO, Kim TS, Kim SK, Kim TH, Kim MC, Park GS, Kim JH, Kuninaka $\mathrm{S}$, Olson EN, et al. (2016) LATS-YAP/TAZ controls lineage specification by regulating TGF $\beta$ signaling and $H n f 4 \alpha$ expression during liver development. Nat Commun 7:11961.

Lin JI, Poon CL, and Harvey KF (2013) The Hippo size control pathway-ever expanding. Sci Signal 6:pe4.

Liu-Chittenden Y, Huang B, Shim JS, Chen Q, Lee SJ, Anders RA, Liu JO, and Pan D (2012) Genetic and pharmacological disruption of the TEAD-YAP complex suppresses the oncogenic activity of YAP. Genes Dev 26:1300-1305.

Maronpot RR, Yoshizawa K, Nyska A, Harada T, Flake G, Mueller G, Singh B, and Ward JM (2010) Hepatic enzyme induction: histopathology. Toxicol Pathol 38: 776-795.

Rodríguez-Antona C, Donato MT, Boobis A, Edwards RJ, Watts PS, Castell JV, and Gómez-Lechón M-J (2002) Cytochrome P450 expression in human hepatocytes and hepatoma cell lines: molecular mechanisms that determine lower expression in cultured cells. Xenobiotica 32:505-520.

Seglen PO (1976) Preparation of isolated rat liver cells. Methods Cell Biol 13:29-83. Shizu R, Abe T, Benoki S, Takahashi M, Kodama S, Miayata M, Matsuzawa A and Yoshinari K (2016) PXR stimulates growth factor-mediated hepatocyte proliferation by cross-talk with the FOXO transcription factor. Biochem $J$ 473: $257-266$

Shizu R, Benoki S, Numakura Y, Kodama S, Miyata M, Yamazoe Y, and Yoshinari K (2013) Xenobiotic-induced hepatocyte proliferation associated with constitutive active/androstane receptor $(\mathrm{CAR})$ or peroxisome proliferator-activated receptor $\alpha$ $(\mathrm{PPAR} \alpha)$ is enhanced by pregnane $\mathrm{X}$ receptor (PXR) activation in mice. PLoS One 8:e61802

Song H, Mak KK, Topol L, Yun K, Hu J, Garrett L, Chen Y, Park O, Chang J, Simpson RM, et al. (2010) Mammalian Mst1 and Mst2 kinases play essential roles in organ size control and tumor suppression. Proc Natl Acad Sci USA 107: 1431-1436

Steinhardt AA, Gayyed MF, Klein AP, Dong J, Maitra A, Pan D, Montgomery EA, and Anders RA (2008) Expression of Yes-associated protein in common solid tumors. Hum Pathol 39:1582-1589.

Tolson AH and Wang H (2010) Regulation of drug-metabolizing enzymes by xenobiotic receptors: PXR and CAR. Adv Drug Deliv Rev 62:1238-1249.

Toriyabe T, Nagata K, Takada T, Aratsu Y, Matsubara T, Yoshinari K, and Yamazoe $Y$ (2009) Unveiling a new essential cis element for the transactivation of the CYP3A4 gene by xenobiotics. Mol Pharmacol 75:677-684.

Tumaneng K, Schlegelmilch K, Russell RC, Yimlamai D, Basnet H, Mahadevan N, Fitamant J, Bardeesy N, Camargo FD, and Guan KL (2012) YAP mediates crosstalk between the Hippo and PI3K-TOR pathways by suppressing PTEN via miR29. Nat Cell Biol 14:1322-1329.

Wang Y, Dong Q, Zhang Q, Li Z, Wang E, and Qiu X (2010) Overexpression of yesassociated protein contributes to progression and poor prognosis of non-small-cell lung cancer. Cancer Sci 101:1279-1285.

Wei P, Zhang J, Egan-Hafley M, Liang S, and Moore DD (2000) The nuclear receptor CAR mediates specific xenobiotic induction of drug metabolism. Nature 407: 920-923.

Williams GM and Whysner J (1996) Epigenetic carcinogens: evaluation and risk assessment. Exp Toxicol Pathol 48:189-195.

Xu MZ, Yao TJ, Lee NP, Ng IO, Chan YT, Zender L, Lowe SW, Poon RT, and Luk JM (2009) Yes-associated protein is an independent prognostic marker in hepatocellular carcinoma. Cancer 115:4576-4585.

Yabuta N, Mukai S, Okamoto A, Okuzaki D, Suzuki H, Torigata K, Yoshida K, Okada $\mathrm{N}$, Miura D, Ito A, et al. (2013) N-terminal truncation of Lats1 causes abnormal cell growth control and chromosomal instability. J Cell Sci 126:508-520.

Yoshinari K, Ohno H, Benoki S, and Yamazoe Y (2012) Constitutive androstane receptor transactivates the hepatic expression of mouse Dhcr24 and human DHCR24 encoding a cholesterogenic enzyme 24-dehydrocholesterol reductase. Toxicol Lett 208:185-191.

Yoshinari K, Takagi S, Yoshimasa T, Sugatani J, and Miwa M (2006) Hepatic CYP3A expression is attenuated in obese mice fed a high-fat diet. Pharm Res 23 $1188-1200$

Yoshinari K, Yoda N, Toriyabe T, and Yamazoe Y (2010) Constitutive androstane receptor transcriptionally activates human $C Y P 1 A 1$ and $C Y P 1 A 2$ genes through a common regulatory element in the 5'-flanking region. Biochem Pharmacol $\mathbf{7 9}$ : 261-269.

Zender L, Spector MS, Xue W, Flemming P, Cordon-Cardo C, Silke J, Fan ST, Luk JM, Wigler M, Hannon GJ, et al. (2006) Identification and validation of oncogenes in liver cancer using an integrative oncogenomic approach. Cell 125:1253-1267. 
Zhang W, Gao Y, Li P, Shi Z, Guo T, Li F, Han X, Feng Y, Zheng C, Wang Z, et al. (2014) VGLL4 functions as a new tumor suppressor in lung cancer by negatively regulating the YAP-TEAD transcriptional complex. Cell Res 24 $331-343$.

Zhang X, George J, Deb S, Degoutin JL, Takano EA, Fox SB, Bowtell DD, and Harvey KF; AOCS Study group (2011) The Hippo pathway transcriptional co-activator, YAP, is an ovarian cancer oncogene. Oncogene 30:2810-2822.

Zhao B, Wei X, Li W, Udan RS, Yang Q, Kim J, Xie J, Ikenoue T, Yu J, Li L, et al (2007) Inactivation of YAP oncoprotein by the Hippo pathway is involved in cell contact inhibition and tissue growth control. Genes Dev 21:2747-2761.
Zhou D, Conrad C, Xia F, Park JS, Payer B, Yin Y, Lauwers GY, Thasler W, Lee JT, Avruch J, et al. (2009) Mst1 and Mst2 maintain hepatocyte quiescence and suppress hepatocellular carcinoma development through inactivation of the Yap1 oncogene. Cancer Cell 16:425-438.

Address correspondence to: Dr. Kouichi Yoshinari, University of Shizuoka, 52-1 Yada, Suruga-ku, Shizuoka, 422-8526, Japan. E-mail: yoshinari@ u-shizuoka-ken.ac.jp 